\title{
La ciencia de la administración pública y la ciencia política en Iberoamérica
}

\author{
González, Juan Antonio*
}

\section{Resumen}

Contiene una reflexión sobre el estatus de la Ciencia de la Administración a partir de la perspectiva de la Ciencia Política, en el marco las preocupaciones sobre la Reforma del Estado y de la Administración Pública. El estudio analiza la producción generada en congresos y reuniones cientificas especializadas realizadas durante el último lustro; aunque ninguna de estas reuniones tuvo por finalidad principal el tratamiento de la Ciencia de la Administración, constituyen un muestrario significativo de la producción académica y científica de lberoamérica referida precisamente a la administración pública y a su objeto principal: el Estado. En cuanto a la Ciencia Política, interesa su relación con la Ciencia de la Administración. Se intenta agrupar las direcciones del cambio planteándolas como rupturas en sus distintos componentes metodológicos y epistemológicos, a fin de apreciar los rasgos comunes y sus orientaciones dominantes. En su diversidad y complejidad, los nuevos enfoques pueden servistos como una verdadera "ruptura epistemológica" en tanto suponen un cambio profundo respecto de los paradigmas que han regido la construcción de la disciplina.

Palabras clave: Ciencia administrativa, ciencia política, paradigma, ruptura epistemológica, reforma de la administración pública.

\section{Fecibido: 98-10-16, Aceptado: 99-02-17}

* Profesor Titular de la cátedra Doctrina e Ideas Políticas Modernas y Contemporáneas. Facultad de Clencias Políticas y Sociales, Universidad Nacional de Cuyo. Argentina. 


\title{
The Science of Public Administration and Political Science in Latin-America
}

\begin{abstract}
This paper contains reflections on the status of the Administrative Sciences from the Political Science perspective, within the framework of State Reform in Public Administration. This study analyzes the production generated in specialized scientific meetings and congresses during the last five years, even though none of these meetings had as its principal objective the study of Administrative Sciences. This constitutes a significant sample of the academic and scientific production in Latin-America with reference to Public Administration and its principal objective: the state. As to Political Science, its relation with Administrative Sciences is very interesting. There is an effort made to group the directions of change proposed as ruptures in their distinct methodological and epistomological components, with the purpose of visualizing common characteristics and dominant orientations. In their diversity and complexity, the new focuses can be seen as true epistomological ruptures in as much as they suggest a profound change with respect to paradigms that have reigned in the constructs of these disciplines.
\end{abstract}

Key words: Administrative science, political science, paradigm, epistomological rupture, public administration reform.

\section{A modo de introducción: Una discusión sobre la pertinencia}

La invitación a reflexionar sobre el estatus de la Ciencia de la Administración en Iberoamérica $y$ especialmente en América Latina a partir de la perspectiva de la Ciencia Política, implica un desafío difícil de afrontar, sobre todo si esta reflexión se realiza en el marco de una convocatoria donde predominan las preocupaciones y planteos específicos sobre la
Reforma del Estado y la Administración Pública. Parece apropiado comenzar por una discusión que contextualice este propósito, tanto en sus alcances como en su pertinencia, respecto del esfuerzo que se lleva a cabo en la comunidad científica latinoamericana, para encontrar respuestas apropiadas y viables a los cambios producidos en el Estado y la sociedad.

Respecto del alcance es necesario acotar, por lo menos, dos dimensiones: la referida al universo en estudio, los casos o fenómenos a observar, y la correspon- 
diente a los enfoques o contenidos que se privilegian en la indagación.

Una consideración exhaustiva de la actividad desarrollada en nuestros países en el campo de la Ciencia de la Administración, debería incluir como mínimo a los centros de estudios tanto de grado como de postgrado, sus curriculum y orientaciones, las unidades y centros de investigación, las instituciones especializadas, las publicaciones generadas por las instituciones y los autores individuales, asi como la producción generada en congresos y reuniones científicas especializadas.

Este nutrido universo requiere para su tratamiento de un esfuerzo y unos medios que sobrepasan largamente las posibilidades de una tarea individual. Obliga por lo tanto a realizar una forzada elección, que limita el alcance de por sí restringido de la presente indagación, a los trabajos presentados en una serie de eventos científicos especializados, a saber:

- II Conferencia Internacional de Ciencias Administrativas, Instituto Internacional de Ciencias Administrativas "Redefinir el perfil del estado en vista de los cambios y desarrollos socioeconómicos" Toluca, México, 1993.

- I Congreso lberoamericano de Ciencia Politica, Organización Iberoamericana de Ciencia Política. "Consolidación democrática y desarrollo socioeconómico en lberoamérica" Santiago de Chile, 1993.

- I Congreso Interamericano del CLAD "Sobre la reforma del Estado y de la administración pública", Río de Janeiro, Brasil, 1996.

- 11 Congreso Interamericano del CLAD "Sobre la reforma del Estado y de la administración pública", Isla de Margarita, Venezuela, 1997

Se puede objetar, con razón, que ninguna de estas reuniones tuvo por finalidad principal el tratamiento de la Ciencia de la Administración. Lo que no se puede negar es que constituyen un muestrario significativo de la producción académica y cientifica de lberoamérica referida precisamente a la administración pública y a su objeto principal: el Estado. La Conferencia del Instituto Internacional de Ciencias Administrativas se incluye por la importante participación que tuvieron en ella las delegaciones de los países hermanos, así como el hecho de que un destacado pensador latinoamericano de la especialidad, el Dr. Bernardo Kliksberg, oficiara como Relator General.

Una mirada desde la perspectiva de la Ciencia Política plantea el problema de resolver previamente los inconvenientes de una disciplina que (también) padece sus propias crisis e indefiniciones, asumida por algunos autores como "crisis de identidad ", pero que en su desarrollo ha consolidado un núcleo de cuestiones que permiten una clara identificación de lo político, "como un campo especifico del proceso social, dedicado a definir y decidir las formas y los objetivos de la vida en común". En todo caso lo que interesa aqui es su relación con la Ciencia de la Administración como cuestión relevante. Puede señalarse como rasgo común con ésta, que el realismo de los estudios politológicos contrasta con la débil institucionalización de la disciplina. (Lechner, 1993).

La discusión sobre la pertinencia de un planteo epistemológico en un marco donde las preocupaciones de los aca- 
démicos e investigadores está centrada en los problemas generados por la magnitud y velocidad de los cambios operados en el Estado y la administración Pública, remite a las preguntas por la oportunidad y la relevancia de esta discusión. ¿Resulta oportuno ocuparse del estatus cientifico de esta disciplina en medio de una realidad que demanda respuestas urgentes? ¿Esta temática es importante para la elaboración de esas respuestas?

El proceso de crisis y reforma del Estado ha generado una profunda movilización en el ámbito de la Administración Pública y este fenómeno, que se percibe como evidente, debiera mirarse como una relación relevante. De pronto estructuras, métodos y paradigmas de las organizaciones públicas que parecían hasta hace pocos años absolutamente cristalizados $y$ hasta inamovibles, se han alterado profundamente y una multitud de nuevos enfoques se han desarrollado e instalado a una velocidad tan acelerada como los cambios sufridos por el Estado. Esta simetría en la respuesta debe sacarse de las reglas o afirmaciones del "sentido común" y ponerse bajo análisis crítico para revisar las relaciones entre la disciplina y su objeto principal, así como su articulación con la ciencia política (llchman, s/f, 55).

Esta situación de cambio pone a los observadores atentos en una posición privilegiada al permitirles ver y comparar a un tiempo lo nuevo y lo viejo, de modo que se hacen patentes sus intimas articulaciones y contradicciones. Luego, cuando la transición haya concluido, y las nuevas fórmulas hayan quedado institucionalizadas, formarán parte del "sentido común" instalado y será mucho más difícil distinguirlas en su originalidad y nove- dad: "Dentro de poco esto será una tarea dificil, pues las revoluciones que triunfan, al hacer desaparecer las causas que las produjeron, resultan incomprensibles debido a su mismo triunfo" (Tocqueville, 1969,29).

La fórmula "la administración cambia porque cambia el Estado" no debe ser puesta en el inventario de los lugares comunes o las verdades de perogrullo: tiene para los contemporáneos de este cambio un valor documental y heuristico que debe mover a un serio compromiso científico y epistemológico con la revisión (reinvención?) de los postulados y métodos de la disciplina. Preguntas tales como si las crisis de la ciencia guardan una relación directa con las crisis de la concepción de Estado, si la administración y sus principios explicativos pueden ser entendidos sin referencia a sus bases sociales y culturales, el papel que juegan los paradigmas cientificos dominantes en la construcción de sus métodos y presupuestos, pueden encontrar respuesta en este marco de reflexión (Guerrero, 1986).

Las crisis crean cierto estado de confusión y desorientación, pero también ofrecen la oportunidad de elevarse por encima de sus componentes accidentales e intentar la incorporación de nuevos ingredientes explicativos, de conceptos y paradigmas que pueden abarcarla y contenerla. Por otro lado, la ciencia es un producto social y puede ser observada como el resultado de la labor de los científicos, expresada en este caso en las reuniones especializadas (Bochenski, 1985,29 y s.s.).

Las ciencias crecen por acumulación de conocimientos sobre sus presupuestos originales, pero también por la 
puesta en discusión y la ampliación de estos presupuestos cuando se muestran insuficientes para explicar la realidad.

Pensar la ciencia en términos de un conjunto de leyes y principios inmutables y definitivos significa una concesión gratuita al idealismo o cuando menos un anacronismo epistemológico del cual las propias "ciencias duras" se han librado hace tiempo (Simard, 1961, 162 y s.s.).

En el caso particular de las ciencias sociales, la revisión de sus presupuestos tiene un interés relevante, ya que sus consecuencias prácticas se proyectan sobre la realidad y producen efectos sobre el Estado y la sociedad.

Se puede establecer un punto e partida: la administración como ciencia social y por tanto como una perspectiva propia con concurrencia metodológica e interdisciplinaria con la política, la sociología, la historia, la sicología, la economia, el derecho.

La cuestión de la crisis de la ciencia de la administración, desde una "una ciencia sin objeto hasta una ciencia sin contenido" puede plantearse entonces como una falta de revisión de sus presupuestos no evidentes, tal como reclama Omar Guerrero respecto de su relación con las clases sociales.

Tampoco puede ser imaginada como un producto atemporal, una suerte de sustancia al modo del idealismo. Esta situación ha sido señalada oportunamente por Bernardo Kliksberg al referirse a los cambios de paradigma de la disciplina, reclamando por una ciencia históricamente situada, con todas las implicancias de una historicidad enriquecida por los datos sociales y culturales.
En lo que se refiere a presupuestos, parece ligada por lo menos a dos marcos de referencia cuya vinculación, por no dilucidada, pueden entrar como componentes de las crisis del objeto y de la disciplina:

- Su vinculación histórica a distintos modelos de organización política, en particular a los diversos modelos de Estado (Absolutista, Demoliberal, Socialista, de Bienestar).

- El predominio del paradigma normativo y una concepción sin matizar de la organización burocrática, tributaria del modelo weberiano.

Hoy estos presupuestos han sido enriquecidos por el aporte pluridisciplinario y transdisciplinario, a los que debería agregarse la perspectiva de la cultura (ya incorporada en algunos análisis organizativos e institucionales), como un marco explicativo y dinamizador más amplio y contenedor.

Si se la considera en su íntima vinculación al Estado y asociada permanentemente a él, no puede ser pensada sin su articulación con la ciencia política como marco natural y propio. Organización, legitimidad, institucionalización, descentralización, poder, liderazgo, relación público privado, participación, control, democratización, sólo para citar algunos de sus temas y contenidos más frecuentes, muestran una línea de continuidad que impiden verla como una ciencia autorreferencial y aislada de su propio contexto ontológico y epistemológico.

Esta perspectiva parece apropiada para resituar la ciencia de la administración en su propio cauce, dirección que al parecer es la que predomina en la actualidad. 
Entonces, una apreciación en términos de los fundamentos de la disciplina debe producirse, no a pesar de les orientaciones y preocupaciones particulares por adaptarse y responder a los cambios, sino precisamente a favor de ellas, porque permiten evidenciar y significar sus articulaciones con las realidades sociales, económicas y políticas que le siven de sustento. Resulta oportuno, por tanto, poner la Biblia de los principios orientadores de la Administración, junto al calefón donde arden las viejas teorías y entran en ebullición los nuevos conceptos y tendencias.

Queda todavia por determinar, si se considera que los argumentos a favor de la pertinencia y relevancia de este propósito han quedado mostrados, cuál será el sentido de la indagación propuesta para pasar revista al estado de la ciencia desde la perspectiva del conocimiento político.

Sería sumamente ilustrativo realizar una apreciación cuantitativa acerca de, por ejemplo, cuáles son los temas y conceptos que predominan y que están más directamente relacionados con los contenidos propios de la ciencia política. Amén del reproche de Sartori $(1991,21)$ "aunque lo puedas medir, si no sabes lo que mides y por qué, tu saber sigue siendo insatisfactorio", queda en pie el propósito original de articular y resituar conceptualmente su relación con la ciencia política. Como lo ha expresado claramente Omar Guerrero (1986,51) "el movimiento de administración científica, que agrupó en la década de los treinta a los principales prosélitos de Taylor y Fayol (...), estuvo tan preocupado por crear una ciencia administrativa general, que arrancó a la
Administración Pública de su campo académico natural: la Ciencia Política".

En la actualidad esta articulación se ha visto revalorizada por un movimiento convergente que tiende a poner al Estado como actor principal, aunque no exclusivo, de la actividad política. Desde el punto de vista de la administración, por un tratamiento prioritario en función de las transformaciones ocurridas, particularmente desde fines de la década del 80 (Oszlak, 1997,17). Desde la política, superando la fragmentación metodológica a que lo había sometido el behaviorismo en boga y recuperando una importante tradición latinoamericana ligada a la consideración del Estado como objeto central de la reflexión socio-política (Ansaldi, 1989,3 y s.s.).

La presente indagación tendrá en cuenta un supuesto cuyos alcances sólo se pueden enunciar aquí: la necesaria articulación de la administración con la ciencia política y sus consecuencias sobre aspectos sustanciales de esta disciplina, referidas a su objeto y situación respecto de la política. Pensar la administración sin la política significa no sólo volver a la dicotomía técnica-política, asignándole un valor puramente instrumental y residual, sino deslegitimar la propia naturaleza de lo público: no se trata solamente de brindar técnicas eficientes para su manejo. Si el Estado "es lo que hace" según una contundente definición de Oszlak (1993), la administración realiza (o niega) los valores que el Estado dice sostener y en consecuencia, es un medio expresivo y operativo del poder. Esta dimensión expresiva es la dimensión de los valores: equidad, justicia, libertad, demo- 
cracia, y de los fines. Resulta casi curioso que los juicios de valor sobre la naturaleza y fines del Estado se realicen sin tener en cuenta esta dimensión expresiva, siendo que la administración es el medio más constante de influencia del Estado sobre la vida cotidiana de los ciudadanos. resulta en todo caso más explicable cuando se reduce la administración a su versión "operativa".

En este contexto, la reflexión habrá de centrarse en dos cuestiones:

- Las relativas a los presupuestos e la Ciencia de la Administración, habida cuenta de que comparte con aquella tanto el ámbito del Estado como el ejercicio del poder público, por to que guardan una estrecha relación ontológica y epistemológica.

- Las concernientes a la valoración que desde la ciencia política se puede realizar sobre las nuevas orientaciones de la disciplina en aquellos puntos que posee una mayor densidad política, en tanto afectan cuestiones relacionadas con la naturaleza del Estado y del régimen político en particular.

Bernardo Kliksberg ha señalado lúcidamente algunos de los "cuellos de botella conceptuales" que caracterizan ciertos estilos y modelos de razonar la administración pública.

Se intenta agrupar las direcciones de ese cambio planteándolas como rupturas en sus distintos componentes metodológicos y epistemológicos. Esta estrategia permite ver con cierta claridad los rasgos comunes e intentar un balance sobre sus orientaciones dominantes. Vistos desde la diversidad y la complejidad de las formulaciones de los especialistas, los nuevos enfoques pueden ser vistos, más allá de sus particularidades, como una verdadera "ruptura epistemológica" en tanto suponen un cambio profundo respecto de los paradigmas que han regido la construcción de la disciplina.

\section{La ruptura epistemológica: crisis del paradigma tradicional por acumulación de rupturas}

Las distintas reuniones cientificas mencionadas como referentes de este trabajo, que cubren el período de un lustro, muestran un rasgo distintivo: su extraordinaria fecundidad. Tanto la diversidad de los temas abordados como las orientaciones con que son tratados, señalan una dirección renovadora que los aleja de la concepción convencional de la administración pública, vista como una materia autorreferenciada y aislada de los hechos políticos y de las fuerzas políticas reales.

Un tratamiento cuidadoso permite encontrar los rasgos comunes expresados en términos de rupturas con aquella concepción, en una enunciación que de ninguna manera pretende ser exhaustiva, pero que permite marcar una clara tendencia en la dirección antes señalada: ruptura del objeto, del sujeto, de la dialéctica público-privado, del paradigma formalista y autorreferencial y ruptura del presupuesto de neutralidad.

Cada una de las "rupturas" mencionadas, se encuentra expresada por diversos participantes en los eventos científicos seleccionados para este estudio durante el último lustro. Su análisis pormenorizado constituye un recorrido aleccio- 
nador imposible de detallar aquí, por lo cual esbozamos a continuación sus rasgos generales.

\subsection{Ruptura del objeto}

Uno de los rasgos más comunes y evidentes lo constituye la reformulación del concepto de Estado. Se ha pasado sin atenuantes de una concepción formalista y puramente organizativa, a una más amplia y abarcativa, destacando especialmente sus relaciones con la sociedad $y$ un tratamiento pormenorizado de sus fines. Las relaciones Estado-sociedad han enriquecido sin excepción las consideraciones sobre la reforma, planteándola en un marco explicativo más amplio que el puramente "administrativo" para analizar sus implicancias sobre el sistema político y social.

Asimismo prevalece la concepción del Estado-comunidad sobre la del Estado-aparato poniendo el acento sobre el nuevo sistema de relaciones tanto ad intra como ad extra del estado. La administración recupera sus primigenias orientaciones hacia el desarrollo social y económico y abre todavía un nuevo espacio en las organizaciones sociales: la gerencia social como un modo de gestión orientado a desarrollar los recursos comunitarios que resultan estratégicos para alcanzar ese desarrollo.

\subsection{Ruptura del sujeto}

El paso del administrado al ciudadano como sujeto destinatario de la administración, implica poner en tela de juicio toda una orientación que legitimaba su acción por referencia a sus exigencias endógenas y no a las demandas y necesi- dades reales de los actores sociales. En forma concomitante, éstos han comenzado a tener protagonismo a través de la participación, como mecanismo destinado a asegurar el efectivo control y la calidad de la gestión estatal.

Conviene señalar de paso el equívoco de considerar al administrado como un cliente, visión derivada de las concepciones privatistas y economicistas de la administración, dado que en la relación cliente-empresa lo que se busca privilegiar es el beneficio de la empresa, cuando en la relación pública lo que legitima tanto el accionar como la existencia misma del Estado es el bienestar de los ciudadanos. En todo caso, la tendencia a recuperar la calidad del servicio como un ingrediente necesario de la actividad administrativa, tiene que ver con esta revalorización del sujeto, así como con una concepción menos autoritaria de la función pública.

La evaluación social de la gestión y la diferenciación de las cualidades de los sujetos sociales destinatarios de las acciones del Estado en términos de equidad, pueden interpretarse en esta línea de construcción del sujeto, en la medida en que introduce diferenciaciones a la hora de diseñary aplicar las políticas. No se trata con un estereotipo de administrado, titular de derechos y sujeto de obligaciones jurídicas universales, sino con personas situadas histórica y socialmente.

\subsection{Ruptura de la dialéctica público-privado}

Por largo tiempo lo público ha sido identificado con lo estatal, con sus notas caracteristicas de legitimidad y coactividad, en tanto que la sociedad aparecia como el 
ámbito de lo privado, como una esfera autónoma y autorregulada, sujeto pasivo de la actividad administrativa. Si bien en el modelo de bienestar se sobreentendía la participación, ésta se caracterizaba por ser corporativa y por lo general no institucionalizada. Frente a esta visión, aparece una dimensión ampliada de lo público (lo público no estatal) caracterizada por el interés social, con diferentes modalidades de gestión. En el caso concreto de las concesiones de servicios, la privatización de lo público aparece limitada tanto por el control estatal al que están sometidas las empresas, como por el valor social que se le asigna a estos servicios.

\subsection{Ruptura del paradigma formalista y autorreferencial}

El paradigma formalista parte de la eficacia per se de la norma y el procedimiento para obtener los resultados esperados, predeterminando la acción de las organizaciones. De allí que su legitimidad provenga del apego a la legalidad.

Frente a esta confianza en las organizaciones estatales aparece la interacción con lo social, que tiene como punto de partida a los actores sociales, sus situaciones y demandas, introduciendo de este modo una legitimidad de eficacia con orientación finalística, antes que procedimental.

El acento puesto en las calidades gerenciales y el liderazgo marca esta tendencia a privilegiar la capacidad de gestión por encima de la estructura y la fórmula burocrática. La eficacia como indicador de la acción administrativa y por ende del gasto público, se presenta como una instancia superadora de la eficiencia como medida del rendimiento de las organizaciones.

\subsection{Ruptura del presupuesto de la neutralidad}

Las referencias expresas al marco de la economía capitalista y a los ingredientes ideológicos que acompañan el proceso de transformación del Estado, en particular a su vertiente "neoliberal", muestran la apertura a una ciencia de la administración más crítica y consciente de los valores realizados por el modelo de Estado que "administra". La definición de la administración por exclusión de la política, relegándola a un papel técnico o instrumental, la privó de su objeto, ocultando la consideración de los valores a los que servía, convirtiéndola en un simple medio cuya eficacia era medida en función de la realización de los fines que le venían predeterminados.

Puede observarse en la actualidad una conciencia más clara de que la politicidad está presente no sólo en la determinación de los fines y objetivos, sino en lo instrumental. O mejor, que los valores también se realizan en lo instrumental ( $y$ por ello deja de serlo), cuando se define el cómo, cuándo y el quién en la aplicación de las decisiones de gobierno.

Los análisis pormenorizados y realistas muestran que no existe ia pretendida neutralidad de los instrumentos, en cuanto su aplicación implica costos, cuya incidencia sobre los actores sociales puede ser medida con variables mucho más significativas que las económicas o fiscales.

Si forman parte de la actividad del Estado y por ende de la política, constituyen distribuciones "autoritativas" de va- 
lor, es decir poder, y por lo tanto contribuyen a determinar preferencias y prioridades entre los sujetos y grupos sociales concretos.

\section{Reflexión final: ciencia de la administración y ciencia política}

Por el lado de la Ciencia Política, si bien se observa simetría en los planteos referidos a la naturaieza de las transformaciones del Estado, no aparece como una preocupación central el tratamiento pormenorizado de la dimensión administrativa; salvo en temas de descentralización y gestión local, tal vez por el predominio de las consideraciones operativas sobre las expresivas. Pero también por una tendencia a soslayar la dimensión administrativa de los temas centrales de la política, lo que podría tomarse como una muestra del estado de desvinculación entra ambas disciplinas.

Lo que resulta particularmente notorio en este sentido, es la tendencia a realizar análisis administrativo a partir de modelos de estado "deseables" en lugar de considerar las situaciones reales de desempeño de la política y el aparato estatal.

Esta posición, que muestra la persistencia de la dicotomía entre política y administración (Falçao Martins, 1997), puede tomarse como referencia para marcar algunas valoraciones de las nuevas tendencias en materia de administración desde la óptica de la ciencia política. que sintéticamente pueden expresarse en las siguientes observaciones:

- Necesidad de atender a las reasignaciones de poder que importan los cambios operados, tanto en las mo- dalidades de gestión como en la estructura del Estado. Si el tratamiento de estos cambios se limita a la dimensión técnica y operativa, sin consideración de su viabilidad política, es probable que no sólo desvirtúen sus contenidos sino también su propia eficacia.

- Falta de consideración del marco de institucionalidad política en que se desempeña el sistema administrativo, particularmente del sistema de justicia y del Parlamento, que definen en buena medida ese marco y tienen una incidencia decisiva en el funcionamiento y la orientación del sistema, como elementos de control y legitimidad del mismo.

- Estrecha relación entre los límites im. puestos por los fines y orientaciones del Estado y la cultura política, con las propuestas de reforma, de modo de no convertirlas en variables independientes del sistema.

- Articulación del proceso de reforma con el marco legal del derecho administrativo.

La conciencia generalizada de que la reforma importa un desplazamiento de los límites entre Estado y sociedad y por tanto, una redefinición del ámbito de to público que hace a la intensidad y extensión del poder estatal, es decir al régimen político, no puede quedar como un dato externo a la práctica y la teoría administrativa. Más bien debe generar una agenda de cuestiones claves entre la reforma "administrativa" y el sistema político, que solicita respuestas integradoras.

Seguramente estas respuestas habrán de tener una incidencia directa sobre los presupuestos de la disciplina ad- 
ministrativa y la formación de administradores públicos.

\section{Bibliografía citada}

Ansaldi, Waldo y Moreno, José Luis (1989) Estado y sociedad en el pensamiento nacional, Cántaro, Buenos Aires.

Bochenski, I.M. (1985) Los métodos actuales del pensamiento, Rialp, Madrid.

Falçao Martins, Humberto (1997) Administración pública gerencial y burocracia. La persistencia de la dicotomía entre política y administración. Reforma y Democracia N 9 , Caracas.

Guerrero, Omar (1986) La administración pública del estado capitalista, Fontamara, México.

Guerrero, Omar (1997) Principios de administración püblica. Escuela Superior de Administración Pública, Bogotá.

Ilchman, Warren (s/f) Administración pública comparativa y el "sentido común académico", compilado por Oscar Oszlak en Teoría de la burocracia estatal: enfoques críticos, Paidós.

Kliksberg, Bernardo (1989) Hacia un nuevo paradigma en gestión pública, Seminario Internacional sobre Redimensionamiento y Modernización de la
Administración Pública en América Latina, INAP, México.

Kliksberg, Bernardo (1994) El rediseño del estado para el desarrollo socioeconómico y el cambio: una agenda para su discusión. Reforma y Democracia, № 2, Caracas.

Lechner, Norberto (1993) Desafíos y perspectivas de la ciencia política, ponencia I Congreso Iberoamericano de Ciencia Política, Santiago de Chile.

Oszlak, Oscar (1993) Estado y sociedad: las nuevas fronteras. Ponencia en el IX Congreso Nacional de Administración Pública, INAP, Mendoza, México.

Oszlak, Oscar (1997, compilación) Estado y sociedad: las nuevas reglas del juego, Colección CEA-CBC, Universidad de Buenos Aires.

Sartori, Giovanni (1991) Hacia donde va la ciencia política. Rev. de Ciencia Politica Vol.XIII №1-2, Santiago de Chile.

Simard, Emile (1961) Naturaleza y alcance del método científico. Gredos, Madrid.

Tocqueville, Alexis de (1969) El antiguo régimen y la revolución, Guadarrama, Madrid. 